\title{
Recurrence of Proliferative Glomerulonephritis with Monoclonal Immunoglobulin G Deposits with a Striated Ultrastructure
}

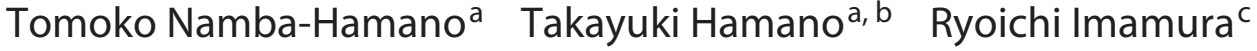 \\ Yutaka Yamaguchi $^{d}$ Masahiro Kyo ${ }^{\text {Hiroaki Yonishi }}{ }^{a}$ Atsushi Takahashi $^{a}$ \\ Masataka Kawamurac Shigeaki Nakazawa ${ }^{c}$ Taigo Katoc ${ }^{c}$ Toyofumi Abe ${ }^{c}$ \\ Miyaji Kyakuno ${ }^{f}$ Yoshitsugu Takabatake $^{a}$ Norio Nonomura $^{c}$ Yoshitaka Isaka $^{a}$ \\ ${ }^{a}$ Department of Nephrology, Osaka University Graduate School of Medicine, Osaka, Japan; bepartment of \\ Nephrology, Nagoya City University Graduate School of Medical Sciences, Nagoya, Japan; 'Department of Urology, \\ Osaka University Graduate School of Medicine, Osaka, Japan; 'Yamaguchi's Pathological Laboratory, Chiba, Japan; \\ 'Osaka Umeda Iseikai Daialysis Clinic, Osaka, Japan; 'Department of Kidney Transplantation, Takatsuki General \\ Hospital, Osaka, Japan
}

\section{Keywords}

Membranoproliferative glomerulonephritis - Proliferative glomerulonephritis with monoclonal lgG deposits .

Recurrent glomerular disease - Renal transplantation .

Striated structure

\begin{abstract}
A 64-year-old man with nephrotic syndrome was admitted to another hospital where his renal biopsy revealed membranoproliferative glomerulonephritis (MPGN) with monoclonal immunoglobulin (lg) G, subclass 1, K light chain (IgG1k) deposition on immunofluorescence (IF). Proliferative glomerulonephritis with monoclonal lgG deposits (PGNMID) was suspected due to monoclonal $\lg \mathrm{G} 1 \mathrm{~K}$ deposits and the absence of hematological abnormalities. However, the typical PGNMID phenotype was not observed by electron microscopy. Instead, an organized and striated muscle-like structure was observed in the subendothelial space. Since a
\end{abstract}

2-year treatment with immunosuppressants did not improve his proteinuria, a second biopsy was performed at our hospital. It showed an MPGN-like phenotype; however, monoclonal Ig deposits on IF were no longer observed. One year after the second biopsy, he developed ESRD. Thus, he underwent living kidney transplantation from his wife. Allograft biopsy was performed as proteinuria was observed 3 months after transplantation, which again showed an MPGN-like phenotype with monoclonal lgG1 $\mathrm{k}$ deposits. The observed electron-dense deposits were similar to those in the native biopsies. Accordingly, the patient was diagnosed with recurrent MPGN. Adding methylprednisolone pulse therapy to conventional immunosuppressants did not improve the patient's renal function or proteinuria. He died of Legionella pneumonia 8 months after transplantation. Considering the patient's histological findings of MPGN with monoclonal $\lg \mathrm{G} 1 \mathrm{~K}$ deposits and early recurrence of glomerulonephritis after transplantation, he was diagnosed with PGNMID with novel electron-dense deposits.

(c) 2020 S. Karger AG, Basel

$\begin{aligned} & \text { karger@karger.com } \\ & \text { www.karger.com/nef }\end{aligned}$
Karger ${ }^{\prime /}$

Tomoko Namba-Hamano

Department of Nephrology, Osaka University Graduate School of Medicine 2-2, Yamada-oka, Suita

Osaka 5650871 (Japan)

namba@kid.med.osaka-u.ac.jp 


\section{Introduction}

Recurrent glomerulonephritis has attracted attention as a cause of graft loss after kidney transplantation in recent advancements in immunosuppressive treatment [1, 2]. Membranoproliferative glomerulonephritis (MPGN) with monoclonal immunoglobulin (Ig) deposits shows a higher recurrence rate after transplantation than MPGN with polyclonal Ig, with subsequent progression to graft loss [3].

Proliferative glomerulonephritis with monoclonal IgG deposits (PGNMID), described by Nasr et al. [4], is characterized by MPGN-like histological findings and glomerular deposition of monoclonal Ig. However, only 10$30 \%$ patients with PGNMID have M protein $[5,6]$. A recent study revealed that PGNMID frequently recurs early after transplantation. We present a case of recurrent PGNMID with a novel and unclassified organized glomerular structure.

\section{Case Report}

\section{Clinical Course of a Native Kidney}

A 64-year-old Japanese man was hospitalized with lower leg edema. Although proteinuria had been noted 3 years before admission, he had not received treatment. No remarkable family history was noted. Upon admission, the patient's blood pressure was 179/119 $\mathrm{mm} \mathrm{Hg}$; urinalysis showed proteinuria of $9.4 \mathrm{~g} / \mathrm{g} \mathrm{Cr}$ and hematuria $(2+)$. Laboratory examination revealed the following: serum $\mathrm{Cr}$, $1.45 \mathrm{mg} / \mathrm{dL}$; total protein, $4.1 \mathrm{~g} / \mathrm{dL}$; albumin, $1.7 \mathrm{~g} / \mathrm{dL}$; low-density lipoprotein, $208 \mathrm{mg} / \mathrm{dL}$; and IgG, $627 \mathrm{mg} / \mathrm{dL}$. Serum complement levels were within the normal ranges. Light microscopy of the first renal biopsy specimen revealed lobular glomerular alterations with mesangial and endocapillary hypercellularity and glomerular basement membrane duplication, typical of MPGN. Immunofluorescence (IF) revealed weak glomerular staining for IgG, IgM, C3, C1q, and fibrinogen, with a peripheral capillary pattern. Subsequent immunostaining for IgG subclasses and light chains revealed mono-

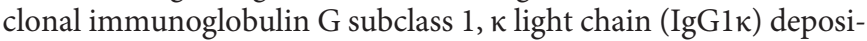

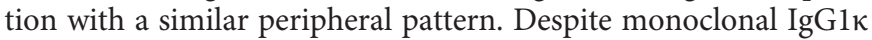
deposition in the glomeruli, M protein was not detected in the blood or urine, and there was no evidence of plasma cell dysplasia. Electron microscopy revealed the accumulation of organized deposits with a striated pattern in the subendothelial space and mesangial area, atypical for MPGN. These electron-dense bands were 10-12$\mathrm{nm}$ wide with a 25-30-nm distance of periodicity. Markers of secondary MPGN, including antinuclear antibody, cryoglobulin, and antibodies for hepatitis B and C, were not detected. The patient was diagnosed with primary MPGN, and oral prednisolone ( $35 \mathrm{mg} /$ day) was administered. Considering that 1 -year immunosuppressive treatment was not effective in improving the patient's proteinuria and renal dysfunction, he was referred to our hospital, and a second biopsy was performed 2 years after the first biopsy.

Findings on the second biopsy were similar to those of the first biopsy, except for IF results; different from the first biopsy, no sig- nificant staining for Ig or complement was observed. The second biopsy specimen included a higher percentage of sclerotic glomeruli (27\%) than the first biopsy specimen (15\%). Moreover, the glomeruli of the second biopsy specimen on IF were more sclerotic compared to those of the first biopsy specimen, and the deposition could not be detected. This probably explains the discrepancy in terms of monoclonal deposition between the first and second biopsies. There was no evidence of a hematological disorder or secondary MPGN. Subsequent 1-year immunosuppressive treatment did not improve patient's renal function, and hemodialysis was initiated.

\section{Clinical Course of the Allograft Kidney}

Two years later, at the age of 68 years, he underwent $A B O$-incompatible living kidney transplantation (blood type $\mathrm{A}$ to $\mathrm{O}$ ) from his 68-year-old wife. For preoperative desensitization, rituximab (200 mg/body) was administered 2 weeks before transplantation, and 2 sessions of plasmapheresis followed by plasma exchange (PE) were performed. Baseline allograft biopsy showed no significant abnormality except for mild arteriosclerosis. A maintenance immunosuppressive regimen included prednisolone, tacrolimus (TAC), and mycophenolate mofetil (MMF). Serum $\mathrm{Cr}$ increased from 1.29 to $2.37 \mathrm{mg} / \mathrm{dL} 3$ weeks after transplantation. The second allograft biopsy, which was performed 3 weeks after transplantation, showed endocapillary proliferation and peritubular capillaritis without mesangial proliferation. Although we found positive staining for $\mathrm{C} 4 \mathrm{~d}$ on the peritubular capillaries on immunohistochemistry, these results might be attributed to $\mathrm{ABO}$-incompatible kidney transplantation. Considering the absence of positive staining for Ig and complements on IF, these findings were consistent with active antibody-mediated rejection. Methylprednisolone pulse therapy and rituximab were added to the maintenance therapy, followed by 2 sessions of PE and $6 \mathrm{~g}$ of intravenous Ig. The additional treatment restored serum $\mathrm{Cr}$ to $1.51 \mathrm{mg} / \mathrm{dL}$ and the patient was discharged. Two months after discharge, his proteinuria level increased to $6.8 \mathrm{~g} / \mathrm{gCr}$ with a slight increase in serum $\mathrm{Cr}$ level to $1.75 \mathrm{mg} / \mathrm{dL}$. Hence, we performed a third allograft biopsy 3 months after transplantation. Light microscopy revealed the absence of sclerotic glomeruli; however, more than half of the 23 glomeruli showed mesangial proliferation with nodules, which were negative for Congo red staining. Endocapillary proliferation and cellular crescents were also observed in some glomeruli, with segmental glomerular basement membrane duplication (Fig. 1). Mild interstitial fibrosis, tubular atrophy, and intimal fibroelastosis were observed, with no evidence of rejection. No Ig or complement was detected on IF-stained frozen tissues. Immunohistochemistry of paraffin-embedded tissues digested with proteinase

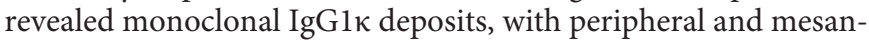
gial patterns (Fig. 2). Electron microscopy revealed a few dense deposits with striated ultrastructure in the subendothelial space and paramesangial area, similar to those observed in the native kidney biopsies (Fig. 3). Accordingly, the patient was diagnosed with recurrent glomerulonephritis. Methylprednisolone pulse therapy was added to maintenance immunosuppressive therapy for the treatment of proteinuria $(4-6 \mathrm{~g} / \mathrm{gCr})$ and kidney disease as evidenced by the elevated serum Cr level of $2.38 \mathrm{mg} / \mathrm{dL}$. At 8 months after transplantation, the patient was admitted following the complaints of fever and dyspnea, and he was diagnosed with Legionella pneumonia. Antibiotics were ineffective, and he subsequently died. 

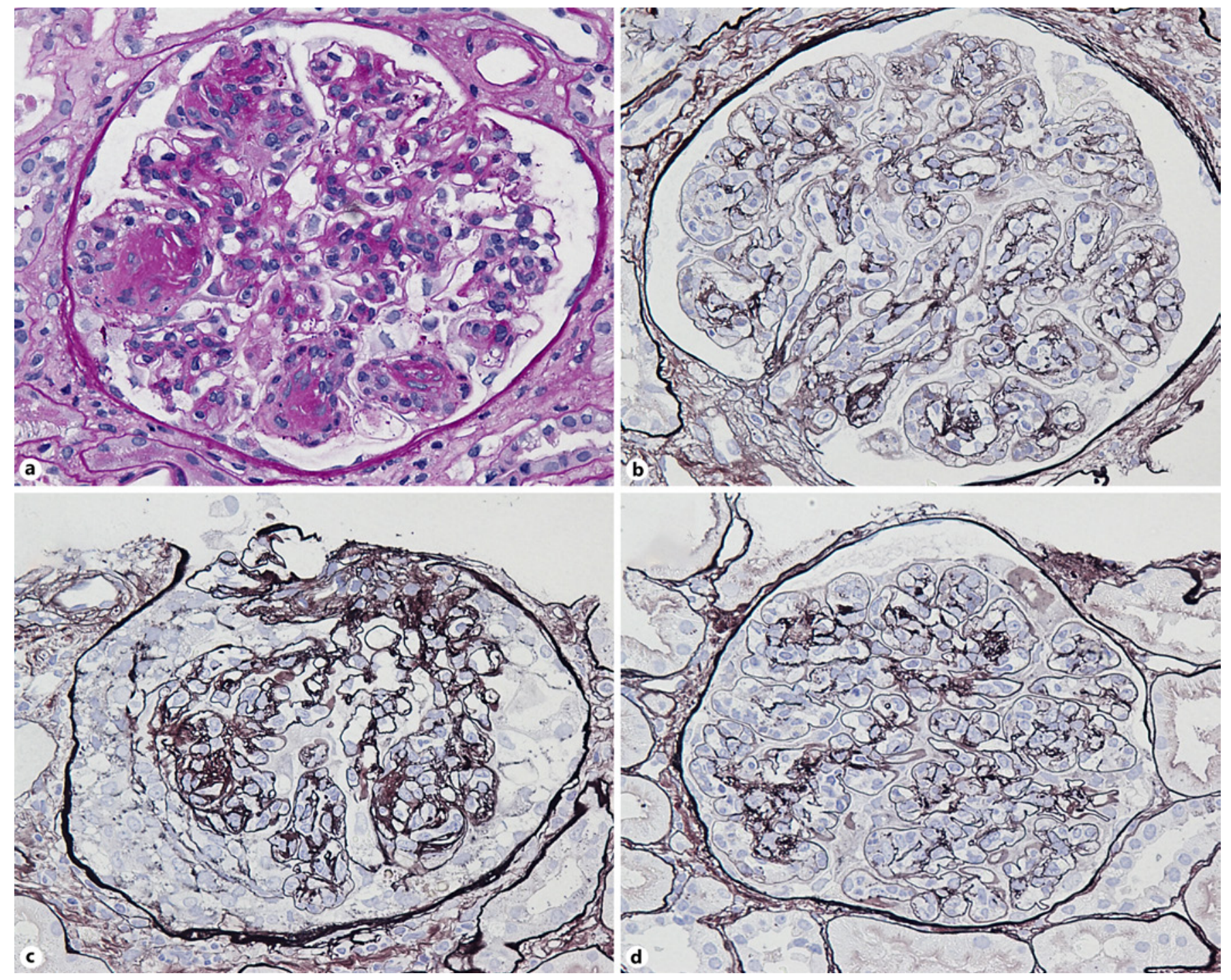

Fig. 1. Light microscopic findings of the third allograft biopsy 3 months after transplantation. a The glomerulus showing mesangial hypercellularity and nodules (periodic acid-Schiff staining. $\times 400$ ). b Double-contour glomerular basement membrane and mesangial interposition (Jones silver methenamine. $\times 400$ ). c, d The glomerulus with a crescent $(\mathbf{c})$ and endocapillary hypercellularity $(\mathbf{d})$ (Jones silver methenamine. $\times 400$ ).

\section{Discussion}

We report a case of novel MPGN with monoclonal Ig deposits and recurrence early after transplantation. Proteinuria was observed 1 month after transplantation, and histological recurrence was confirmed with a protocol biopsy performed 3 months later.

Based on the pathological features of MPGN and monoclonal Ig deposits in the glomeruli, with the absence of $\mathrm{M}$ protein, this case met the criteria for PGNMID described by Nasr et al. [4]. Features observed on electron microscopy typical of PGNMID include unorganized granular deposits localized to the subendothelial space, the mesangial area, and/or the subepithelial space. The clinical outcomes are variable, with approximately $25 \%$ of patients developing ESRD refractory to immunosuppressive treatment within 2.5 years [7]. A recent study of protocol biopsies revealed that PGNMID had a high recurrence rate (89\%) early after transplantation. In this study, approximately half of the patients lost graft function within 3 years after diagnosis [6]. Kawanishi et al. reported a case of recurrent PGNMID leading to rapid graft loss 


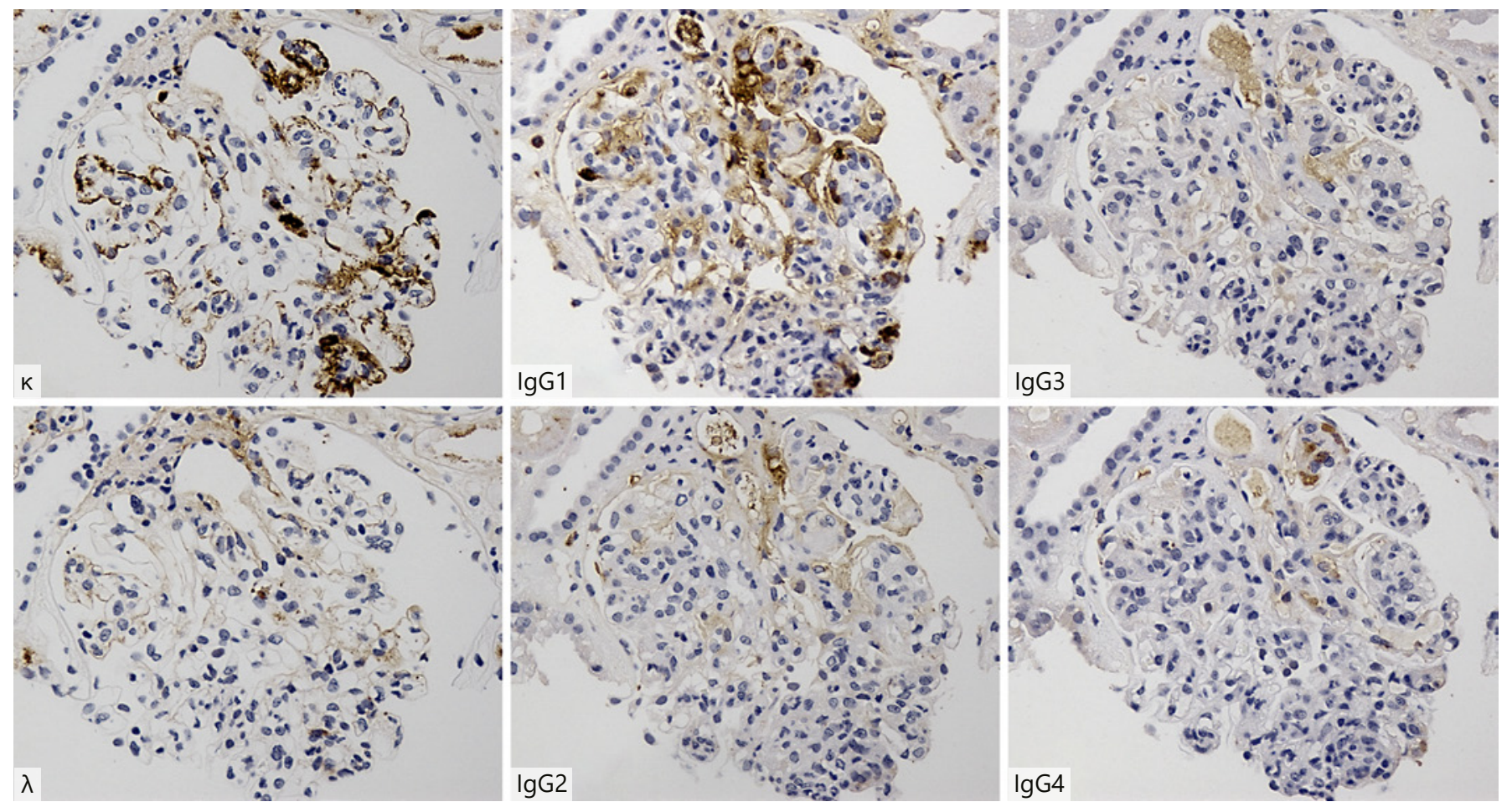

Fig. 2. Immunohistochemistry of paraffin-embedded tissues digested with proteinase for the third allograft biopsy specimens 3 months after transplantation. Monoclonal immunoglobulin $G$, subclass 1 , $\kappa$ light chain deposits with peripheral and mesangial pattern $(\times 400)$.

after transplantation [8]. Considering a high recurrence rate and poor prognosis, careful consideration is required to perform kidney transplantation for patients progressing to ESRD due to PGNMID.

The presence of monoclonal Ig deposits in the kidney is highly suggestive of the presence of underlying B-cell or plasma cell clones. Renal diseases with monoclonal Ig are likely to recur after transplantation due to impaired eradication of small B-cell clones. PGNMID has a shorter time to recurrence than light chain amyloidosis and monoclonal Ig deposition disease (MIDD). Despite monoclonal Ig deposits in the kidney, repeated hematological evaluations failed to detect significant abnormalities. Only $10-30 \%$ patients with PGNMID had detectable $M$ protein in the serum and urine electrophoresis (EP) or immunofixation $[5,6]$. A possible explanation is that the amount of monoclonal Ig was significantly small for detection using the serum or urine EP due to a few underlying B-cell clones.

This case showed unusually organized deposits exclusively in the glomeruli on electron microscopy. The deposits contained regularly stacked electron-dense bands in a parallel arrangement, resembling striated myofibrils.
Organized patterns of fibrils, microtubules, and granular deposits have typically been observed in renal diseases [9]. The striated structure observed in the present case was different and could not be conventionally classified. Although the present case showed ultrastructure similar to fibrin tactoids, its periodicity $(25-30 \mathrm{~nm})$ was slightly longer than that of fibrin tactoids $(22-23 \mathrm{~nm})$ [10]. Some studies have reported cases of renal disease with similar ultrastructure $[11,12]$. The patient's medical story before transplantation in this present case report was summarized in another article [13]. There was no evidence of systemic disease such as cryoglobulinemia, except for 1 case with multiple myeloma. On light microscopy, all cases showed similar features, with negative Congo red staining results and MPGN-like findings. The IF results varied. Some cases were positive for $\mathrm{C} 3$ and $\mathrm{C} 1 \mathrm{q}$, while others showed no positive findings.

The present case showed similar electron-dense deposits with a striated ultrastructure, but 2 unique features were observed. First, this patient relapsed after $\mathrm{ABO}$-incompatible transplantation, suggesting pathology refractory to conventional immunosuppressants including TAC and MMF, as well as to rituximab and PE, 

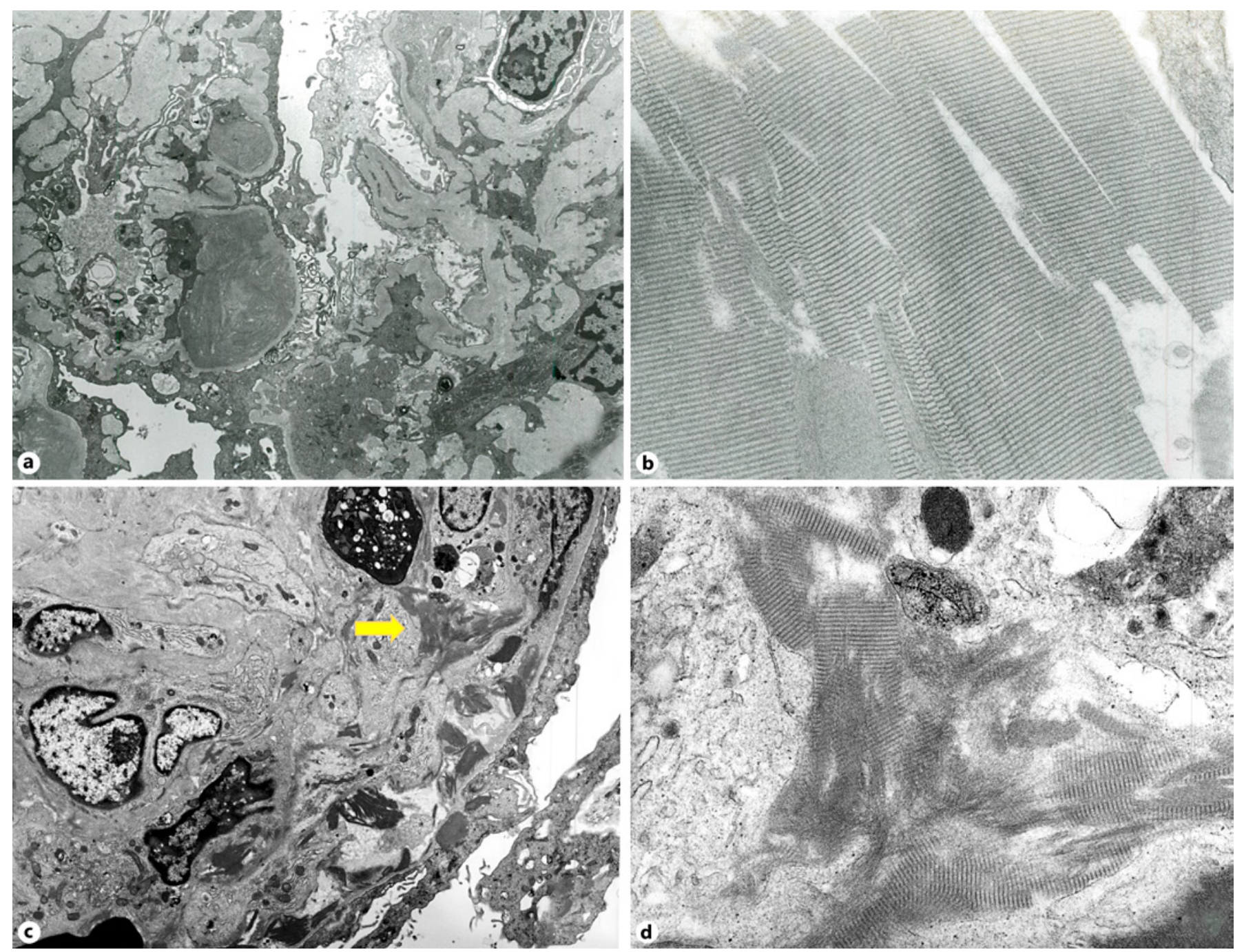

Fig. 3. Electron microscopic findings of the second biopsy of the native kidney (a, b) and the third allograft biopsy 3 months after transplantation (c, d). a Electron-dense deposits accumulated in the subendothelial space $(\times 3,000)$. b Higher magnification of deposits showing striated myofibril-like ultrastructure $(\times 20,000)$. c Some electron-dense deposits (arrow) were observed in the sub-

although a recent study reported the efficacy of clonedirected therapy that included rituximab [14]. Second, we found monoclonal deposits using antigen retrieval. Conventional IF was negative for Igs and complements. However, IgG1 $\kappa$ deposition was unmasked on immunohistochemistry by protease digestion using paraffin-embedded formalin-fixed tissue. Similar false-negative cases were reported in studies showing the usefulness of antigen retrieval [15]. In cases of MPGN negative for Ig on IF, unmasking by antigen retrieval should be considered.

endothelial space in the allograft biopsy $(\times 3,000)$. $\mathbf{d}$ Higher magnification of deposits in the allograft biopsy specimen, similar to those in the native kidney $(\times 15,000)$. The width and distance of periodicity of these electron-dense bands were approximately $10-$ 12 and $25-30 \mathrm{~nm}$, respectively.

A case of PGNMID with another atypical organized deposit was reported [16]. The appearance of the deposits in this case was microlamellar, which was different from the striated muscle-like structure observed in the present case. Considering the definition of PGNMID by Nasr et al. [4], cases with unusual deposits can be diagnosed as atypical PGNMID. Further investigation is needed to elucidate the identity of these deposits.

In conclusion, we report a case of recurrent PGNMID with atypical electron-dense deposits. This is the first report of early recurrence of atypical PGNMID with a stri- 
ated ultrastructure after transplantation. To detect monoclonal Ig deposits with negative staining on IF, immunohistochemistry of paraffin-embedded tissues digested with proteinase was effective.

\section{Acknowledgement}

We thank Drs. S. Hara at Kobe City Medical Center General Hospital and H. Tsukaguchi at Kansai Medical University for providing us with information on the first biopsy. We also thank $\mathrm{Mr}$. S. Horita at Tokyo Women's Medical University Hospital for his technical assistance.

\section{Statement of Ethics}

Written informed consent of the patient for publication has been obtained.

\section{Conflict of Interest Statement}

The authors have no conflict of interest to declare.

\section{Funding Sources}

This work was not supported by any funding sources.

\section{Author Contributions}

T.N.-H. and T.H. drafted and revised the manuscript. R.I., A.T., M.K., S.N., T.K., T.A., M.K., and Y.T. saw the patient directly. Y.Y. and M.K. scrutinized renal biopsy samples. N.N. and Y.I. provided critical review, advice, and consultation throughout the writing of the manuscript.

\section{References}

1 Briganti EM, Russ GR, McNeil JJ, Atkins RC, Chadban SJ. Risk of renal allograft loss from recurrent glomerulonephritis. $\mathrm{N}$ Engl J Med. 2002 Jul;347(2):103-9.

2 Floege J. Recurrent glomerulonephritis following renal transplantation: an update. Nephrol Dial Transplant. 2003 Jul;18(7): $1260-5$.

3 Cosio FG, Cattran DC. Recent advances in our understanding of recurrent primary glomerulonephritis after kidney transplantation. Kidney Int. 2017 Feb;91(2):304-14.

4 Nasr SH, Markowitz GS, Stokes MB, Seshan SV, Valderrama E, Appel GB, et al. Proliferative glomerulonephritis with monoclonal IgG deposits: a distinct entity mimicking immune-complex glomerulonephritis. Kidney Int. 2004 Jan;65(1):85-96.

5 Bridoux F, Leung N, Hutchison CA, Touchard G, Sethi S, Fermand JP, et al. Diagnosis of monoclonal gammopathy of renal significance. Kidney Int. 2015 Apr;87(4):698-711.

6 Said SM, Cosio FG, Valeri AM, Leung N, Sethi S, Salameh H, et al. Proliferative glomerulonephritis with monoclonal immunoglobulin $\mathrm{G}$ deposits is associated with high rate of early recurrence in the allograft. Kidney Int. 2018 Jul;94(1):159-69.
7 Nasr SH, Satoskar A, Markowitz GS, Valeri AM, Appel GB, Stokes MB, et al. Proliferative glomerulonephritis with monoclonal IgG deposits. J Am Soc Nephrol. 2009 Sep;20(9): 2055-64.

8 Kawanishi K, Honda K, Horita S, Koike J, Shimizu T, Tanabe K, et al. Recurrent proliferative glomerulonephritis with monoclonal immunoglobulin $\mathrm{G}$ deposits leads to rapid graft loss after kidney transplantation: a case report. CEN Case Rep. 2014 Nov;3(2):139-44.

9 Herrera GA, Turbat-Herrera EA. Renal diseases with organized deposits: an algorithmic approach to classification and clinicopathologic diagnosis. Arch Pathol Lab Med. 2010 Apr;134(4):512-31.

10 Iskandar SS, Herrera GA. Glomerular diseases with organized deposits. In: Charles Janette J, Olson JL, Silva FG, D'Agati VD, editors. Heptinstall's pathology of the kidney. Philadelphia, PA: Lippincott Williams \& Wilkins (LWW); 2014. Vol. 2; p. 1015-39.

11 Ohtani H, Wakui H, Komatsuda A, Goto H, Tada M, Ozawa M, et al. Progressive glomerulopathy with unusual deposits of striated structures: a new disease entity? Nephrol Dial Transplant. 2010 Jun;25(6):2016-9.
12 Lee YG, Shin DY, Lee HJ, Choi SY, Park JY, Ahn SY, et al. Proliferative glomerulopathy with unusual subendothelial deposits of striated structure in multiple myeloma. Korean J Nephrol. 2010;29(6):772-5.

13 Hara S, Tsukaguchi H, Oka T, Kusabe M Mizui M, Joh K. Monoclonal immunoglobulin-associated proliferative glomerulonephritis characterized by organized deposits of striated ultra-substructures: a case report. Ultrastruct Pathol. 2017 Jul-Aug;41(4):301-7.

14 Gumber R, Cohen JB, Palmer MB, Kobrin SM, Vogl DT, Wasserstein AG, et al. A clonedirected approach may improve diagnosis and treatment of proliferative glomerulonephritis with monoclonal immunoglobulin deposits. Kidney Int. 2018 Jul;94(1):199-205.

15 Larsen CP, Messias NC, Walker PD, Fidler ME, Cornell LD, Hernandez LH, et al. Membranoproliferative glomerulonephritis with masked monotypic immunoglobulin deposits. Kidney Int. 2015 Oct;88(4):867-73.

16 Mii A, Shimizu A, Takada D, Tsuruoka S. Proliferative glomerulonephritis with unusual microlamellar organized deposits related to monoclonal immunoglobulin G3 (IgG3) kappa. CEN Case Rep. 2018 Nov;7(2):320-4. 